\title{
Almost periodic solutions for a nonlinear integro-differential equation with neutral delay
}

Qiu-Feng Zou, Hui-Sheng Ding*

College of Mathematics and Information Science, Jiangxi Normal University, Nanchang, Jiangxi 330022, P. R. China.

Communicated by N. Shahzad

\begin{abstract}
This paper is concerned with the existence of almost periodic and pseudo almost solutions for a nonlinear integro-differential equation with neutral delay, which arise in epidemic problems. By using almost periodic functions theory and fixed point theory, we obtain the results. Two examples are given to illustrate our results. In addition, an application to nonautonomous differential equations is also given. (C)2016 All rights reserved.
\end{abstract}

Keywords: Almost periodic, pseudo almost periodic, integro-differential, neutral delay.

2010 MSC: 45G10, 34K14.

\section{Introduction and preliminaries}

In 1990, Fink and Gatica [12] firstly studied the existence of positive almost periodic solution to the following delay integral equation

$$
x(t)=\int_{t-\tau}^{t} f(s, x(s)) d s, \quad t \in \mathbb{R},
$$

which is a model for the spread of some infectious disease (cf. [15, 17]). Since then, the existence of positive almost periodic type solution to equation (1.1) and its variants has been of great interest for many authors (see, e.g., 116, 8, 11, 14, 16 and references therein).

In 1997, Ait Dads and Ezzinbi [4] considered the existence of positive almost periodic solutions for the following neutral integral equation

$$
x(t)=\gamma x(t-\tau)+(1-\gamma) \int_{t-\tau}^{t} f(s, x(s)) d s,
$$

\footnotetext{
*Corresponding author

Email addresses: 642074260@qq.com (Qiu-Feng Zou), dinghs@mail.ustc.edu.cn (Hui-Sheng Ding)
} 
and, in 2009, Ait Dads, Cieutat and Lhachimi [1] discussed the existence of positive pseudo almost periodic solution to the following more general infinite delay integral equation

$$
x(t)=\int_{-\infty}^{t} a(t, t-s) f(s, x(s)) d s
$$

which unifies equation (1.1) and equation 1.2. Afterwards, in 2001, Ding, Chen and N'Guérékata [8] investigated the existence of positive pseudo almost periodic solution to the following neutral integral equation:

$$
x(t)=\alpha(t) x(t-\beta)+\int_{-\infty}^{t} a(t, t-s) f(s, x(s)) d s+h(t, x(t)) .
$$

Very recently, by using Perov's fixed point theorem, Bellour and Ait Dads [5] studied the existence and uniqueness of periodic solutions for the following nonlinear integro-differential equation with neutral delay:

$$
x(t)=\gamma x(t-\sigma(t))+(1-\gamma) \int_{t-\sigma(t)}^{t} f\left(s, x(s), x^{\prime}(s)\right) d s, \quad t \in \mathbb{R} .
$$

As noted in [5], Eq. (1.4) includes many important integral and functional equations that arise in biomathematics.

Stimulated by the above works, in this paper, we aim to make further study on this topic, i.e., we will investigate the existence of almost periodic and pseudo almost solutions for the following integro-differential equation with neutral delay:

$$
x(t)=\alpha(t) x(t-\sigma(t))+\int_{-\infty}^{t} \beta(t, t-s) f\left(s, x(s), x^{\prime}(s)\right) d s, \quad t \in \mathbb{R} .
$$

Throughout the rest of this paper, we denote by $\mathbb{R}$ the set of real numbers, by $\mathbb{R}^{+}$the set of nonnegative real numbers, by $X$ a Banach space, by $\Omega$ a subset of $\mathbb{R}^{n}$. Moreover, $C(\mathbb{R})$ (respectively $C(\mathbb{R} \times \Omega)$ ) denotes the space of real-valued bounded continuous functions $f(t)$ (res. $f(t, x)$ ) from $\mathbb{R}($ res. $\mathbb{R} \times \Omega$ ) to $\mathbb{R}$ with norm $\|f\|=\sup _{t \in \mathbb{R}}|f(t)|\left(\right.$ res. $\left.\|f\|=\sup _{t \in \mathbb{R}, x \in \Omega}|f(t)|\right) ; C^{n}(\mathbb{R})$ denotes the space of all functions which have a continuous $n-t h$ derivative on $\mathbb{R}$, with the norm $\|f\|^{n}=\sum_{i=0}^{n} \sup _{t \in \mathbb{R}}\left|f^{(i)}(t)\right|$, where $f^{(i)}$ denote the $i-t h$ derivative of $f$ and $f^{(0)}=f, f^{(1)}=f^{\prime}$.

Next, let us recall some basic notations and properties about almost periodic functions and pseudo almost periodic functions. For more details, we refer the reader to [18].

Definition 1.1. A continuous function $f: \mathbb{R} \rightarrow X$ is called almost periodic if for every $\varepsilon>0$ there exists $l(\varepsilon)>0$ such that every interval $I$ of length $l(\varepsilon)$ contains a number $\tau$ with the property that

$$
\|f(t+\tau)-f(t)\|<\varepsilon \text { for all } \mathrm{t} \in \mathbb{R} .
$$

We denote by $A P(X)$ the set of all such functions.

Definition 1.2. A continuous function $f: \mathbb{R} \times \Omega \rightarrow X$ is called almost periodic in $t$ uniformly for $x \in \Omega$ if for every $\varepsilon>0$ and for every compact subset $K \subset \Omega$ there exists $l(\varepsilon)>0$ such that every interval $I$ of length $l(\varepsilon)$ contains a number $\tau$ with the property that

$$
\|f(t+\tau, x)-f(t, x)\|<\varepsilon \quad \text { for all } \mathrm{t} \in \mathbb{R}, x \in K .
$$

We denote by $A P(\mathbb{R} \times \Omega, X)$ the set of all such functions.

Let

$$
P A P_{0}(X)=\left\{f \in B C(\mathbb{R}, X): \lim _{r \rightarrow+\infty} \frac{1}{2 r} \int_{-r}^{r}\|f(t)\| d t=0\right\}
$$


Similarly, $P A P_{0}(\mathbb{R} \times \Omega, X)$ denotes the collection of all jointly continuous functions $f: \mathbb{R} \times \Omega \rightarrow \mathbb{R}$ such that for every compact subset $K \subset \Omega, f$ is bounded on $\mathbb{R} \times K$, and

$$
\lim _{r \rightarrow+\infty} \frac{1}{2 r} \int_{-r}^{r}\|f(t, x)\| d t=0 \quad \text { uniformly for } x \in K .
$$

Definition 1.3. A function $f: \mathbb{R} \rightarrow X$ (respectively $\mathrm{f}: \mathbb{R} \times \Omega \rightarrow X$ ) is called pseudo almost periodic (respectively pseudo almost periodic in $t$ uniformly with respect to $x \in \Omega$ ) if

$$
f=g+\phi,
$$

where $g \in A P(\mathbb{R})\left(\right.$ respectively $g \in A P(\mathbb{R} \times \Omega, \mathbb{R})$ ) and $\phi \in P A P_{0}(\mathbb{R})\left(\right.$ respectively $\phi \in P A P_{0}(\mathbb{R} \times \Omega, \mathbb{R})$ ). We denote by $P A P(X)$ (respectively $P A P(\mathbb{R} \times \Omega, X)$ ) the set of all such functions.

Lemma 1.4. Let $E \in\{A P(\mathbb{R}), P A P(\mathbb{R})\}$. Then, the following hold true:

(a) $f, g \in E$ implies that $f+g \in E$;

(b) $f, g \in E$ implies that $f \cdot g \in E$;

(c) $f \in E$ implies that $f(\cdot-c) \in E$ for any constant $c \in \mathbb{R}$;

(d) E is a Banach space equipped with the supremum norm.

Lemma 1.5. Let $x, \sigma \in A P(\mathbb{R})$. Then $x(\cdot-\sigma(\cdot)) \in A P(\mathbb{R})$.

Suppose that $H(t) \in \Omega$ for all $t \in \mathbb{R}$. Define $H \times \iota: \mathbb{R} \rightarrow \Omega \times \mathbb{R}$ by

$$
H \times \iota(t)=\left(t, h_{1}(t), h_{2}(t) \cdots h_{n}(t)\right), \quad t \in \mathbb{R} .
$$

Lemma 1.6. Let $f \in A P(\mathbb{R} \times \Omega, \mathbb{R}), H \in A P(\mathbb{R})^{n}$ with $H(t) \in \Omega$ for all $t \in \mathbb{R}$. Then $f \circ(H \times \iota) \in A P(\mathbb{R})$.

Lemma 1.7. Let $f \in P A P(\Omega \times \mathbb{R}, \mathbb{R})$ be continuous on every compact subset $K \subset \Omega$ uniformly for $t \in \mathbb{R}$ and $H \in P A P(\mathbb{R})^{n}$ with $H(t) \in \Omega$ for all $t \in \mathbb{R}$. Then $f \circ(H \times \iota) \in P A P(\mathbb{R})$.

Now, let us recall the notion of $C^{n}$-almost periodicity (for more details, we refer the reader to [6] and [9]).

Definition 1.8. A continuous function $f \in C^{n}(\mathbb{R}, \mathbb{R})$ is called $C^{n}$-almost periodic if for every $\varepsilon>0$ there exists $l(\varepsilon)>0$ such that every interval $I$ of length $l(\varepsilon)$ contains a number $\tau$ with the property that

$$
\|f(t+\tau)-f(t)\|^{n}<\varepsilon \text { for all } \mathrm{t} \in \mathbb{R} .
$$

We denote by $A P^{n}(\mathbb{R})$ the set of all such functions.

Remark 1.9. By [6], $A P^{n}(\mathbb{R})$ turns out to be a Banach space equipped with the norm $\|\cdot\|^{n}=\sup _{t \in \mathbb{R}} \sum_{i=0}^{n}\left|f^{(i)}(t)\right|$.

\section{Almost periodic case}

In this section, we study the existence and uniqueness of almost periodic solution for Eq. (1.5). For convenience, we will work on the following norm:

$$
\|f\|_{1}=\|f\|+\left\|f^{\prime}\right\| .
$$

In fact, the two norms are equivalent.

Lemma 2.1. $\left(A P^{1}(\mathbb{R}),\|\cdot\|_{1}\right)$ is a Banach space. 
Proof. By Remark 1.9 , we know that $A P^{1}(\mathbb{R})$ turns out to be a Banach space equipped with the norm $\|f\|^{1}=\sup _{t \in \mathbb{R}}\left(|f(t)|+\left|f^{\prime}(t)\right|\right)$. Noting that

$$
\sup _{t \in \mathbb{R}}\left(|f(t)|+\left|f^{\prime}(t)\right|\right) \leq \sup _{t \in \mathbb{R}}|f(t)|+\sup _{t \in \mathbb{R}}\left|f^{\prime}(t)\right| \leq 2 \sup _{t \in \mathbb{R}}\left(|f(t)|+\left|f^{\prime}(t)\right|\right) .
$$

We have $\|\cdot\|^{1} \leq\|\cdot\|_{1} \leq 2\|\cdot\|^{1}$.

Lemma $2.2([1])$. Let $\beta: \mathbb{R} \times \mathbb{R}^{+} \rightarrow \mathbb{R}$ such that $t \rightarrow \beta(t, \cdot)$ is in $A P\left(L^{1}\left(\mathbb{R}^{+}\right)\right), f \in A P(\mathbb{R})$, and

$$
F(t)=\int_{-\infty}^{t} \beta(t, t-s) f(s) d s, \quad t \in \mathbb{R} .
$$

Then $F \in A P(\mathbb{R})$.

We list some assumptions:

(H1) $\alpha, \sigma \in A P^{1}(\mathbb{R}), f \in A P\left(\mathbb{R} \times \mathbb{R}^{2}, \mathbb{R}\right)$, and $\beta: \mathbb{R} \times \mathbb{R}^{+} \rightarrow \mathbb{R}$ satisfies $\beta(\cdot, 0) \in A P(\mathbb{R})$ and $t \rightarrow \beta(t, \cdot)$ is in $A P\left(L^{1}\left(\mathbb{R}^{+}\right)\right)$.

(H2) $\beta \in C^{1}\left(\mathbb{R} \times \mathbb{R}^{+}, \mathbb{R}\right)$ and there exist $\gamma, \gamma_{1}, \gamma_{2} \in L^{1}\left(\mathbb{R}^{+}\right) \cap C\left(\mathbb{R}^{+}\right)$such that for all $t \in \mathbb{R}$ and a.e. $s \in \mathbb{R}^{+}$, there holds

$$
|\beta(t, s)| \leq \gamma(s), \quad\left|\beta_{1}(t, s)\right| \leq \gamma_{1}(s), \quad\left|\beta_{2}(t, s)\right| \leq \gamma_{2}(s),
$$

where $\beta_{1}(t, s)=\frac{\partial \beta(t, s)}{\partial t}$ and $\beta_{2}(t, s)=\frac{\partial \beta(t, s)}{\partial s}$. Moreover, $t \rightarrow \beta_{i}(t, \cdot), i=1,2$, are both in $A P\left(L^{1}\left(\mathbb{R}^{+}\right)\right)$.

(H3) There exist $L_{f}>0$ such that

$$
|f(t, u, v)|-\left|f\left(t, u^{\prime}, v^{\prime}\right)\right| \leq L_{f}\left(\left|u-u^{\prime}\right|+\left|v-v^{\prime}\right|\right), \quad t, u, u^{\prime}, v, v^{\prime} \in \mathbb{R} .
$$

Theorem 2.3. Assume that (H1)-(H3) hold, and

$$
\|\alpha\|+\left\|\alpha^{\prime}\right\|+\|\alpha\| \cdot\left\|1-\sigma^{\prime}\right\|+\left[\gamma(0)+\|\gamma\|_{L^{1}\left(\mathbb{R}^{+}\right)}+\left\|\gamma_{1}\right\|_{L^{1}\left(\mathbb{R}^{+}\right)}+\left\|\gamma_{2}\right\|_{L^{1}\left(\mathbb{R}^{+}\right)} L_{f}<1 .\right.
$$

Then Eq. 1.5 has a unique almost periodic solution.

Proof. Define $T: A P^{1}(\mathbb{R}) \rightarrow C(\mathbb{R})$ by

$$
T x(t)=\alpha(t) x(t-\sigma(t))+\int_{-\infty}^{t} \beta(t, t-s) f\left(s, x(s), x^{\prime}(s)\right) d s, t \in \mathbb{R} .
$$

We claim that $T$ maps $A P^{1}(\mathbb{R})$ to $C^{1}(\mathbb{R})$, in fact, by (H2), one can obtain

$$
\begin{aligned}
(T x)^{\prime}(t)= & \alpha^{\prime}(t) x(t-\sigma(t))+\alpha(t)\left(1-\sigma^{\prime}(t)\right) x^{\prime}(t-\sigma(t))+\beta(t, 0) f\left(t, x(t), x^{\prime}(t)\right) \\
& +\int_{-\infty}^{t} \beta_{1}(t, t-s) f\left(s, x(s), x^{\prime}(s)\right) d s+\int_{-\infty}^{t} \beta_{2}(t, t-s) f\left(s, x(s), x^{\prime}(s)\right) d s .
\end{aligned}
$$

Let $x \in A P^{1}(\mathbb{R})$. By Lemma 1.5 , we obtain

$$
x(\cdot-\sigma(\cdot)), x^{\prime}(\cdot-\sigma(\cdot)) \in A P(\mathbb{R}) .
$$

Note that $f \in A P^{1}\left(\mathbb{R} \times \mathbb{R}^{2}, \mathbb{R}\right)$, by Lemma 1.6 , we know that

$$
f\left(\cdot, x(\cdot), x^{\prime}(\cdot)\right) \in A P(\mathbb{R}) .
$$


Combining with Lemma 2.2, we obtain

$$
t \rightarrow \int_{-\infty}^{t} \beta(t, t-s) f\left(s, x(s), x^{\prime}(s)\right) d s
$$

is in $A P(\mathbb{R})$. Then, it follows from Lemma 1.4 that $T x \in A P(\mathbb{R})$.

Again by Lemma 1.4 and (H1), we have

$$
t \rightarrow \alpha^{\prime}(t) x(t-\sigma(t))+\alpha(t)\left(1-\sigma^{\prime}(t)\right) x^{\prime}(t-\sigma(t))+\beta(t, 0) f\left(t, x(t), x^{\prime}(t)\right)
$$

belongs to $A P(\mathbb{R})$. Noting that $t \rightarrow \beta_{i}(t, \cdot), i=1,2$, are both in $A P\left(L^{1}\left(\mathbb{R}^{+}\right)\right)$, again by Lemma 2.2 , we conclude that

$$
t \rightarrow \int_{-\infty}^{t} \beta_{i}(t, t-s) f\left(s, x(s), x^{\prime}(s)\right) d s, i=1,2,
$$

are both in $A P(\mathbb{R})$. Thus, $(T x)^{\prime} \in A P(\mathbb{R})$. In conclusion, $T x \in A P^{1}(\mathbb{R})$, which means $T\left(A P^{1}(\mathbb{R})\right) \subset$ $A P^{1}(\mathbb{R})$.

For all $x, y \in A P^{1}(\mathbb{R})$, we have

$$
\begin{aligned}
\|T x-T y\|_{1}= & \|T x-T y\|+\left\|(T x)^{\prime}-(T y)^{\prime}\right\| \\
\leq & \|\alpha\| \cdot\|x-y\|+\sup _{t \in \mathbb{R}} \int_{-\infty}^{t} \gamma(t-s) L_{f}\|x-y\|_{1}+\left\|\alpha^{\prime}\right\| \cdot\|x-y\|+\|\alpha\| \cdot\left\|1-\sigma^{\prime}\right\| \cdot\left\|x^{\prime}-y^{\prime}\right\| \\
& +\gamma(0) L_{f}\|x-y\|_{1}+\sup _{t \in \mathbb{R}} \int_{-\infty}^{t} \gamma_{1}(t-s) L_{f}\|x-y\|_{1}+\sup _{t \in \mathbb{R}} \int_{-\infty}^{t} \gamma_{2}(t-s) L_{f}\|x-y\|_{1} \\
\leq & \left(\|\alpha\|+\left\|\alpha^{\prime}\right\|\right) \cdot\|x-y\|+\|\alpha\| \cdot\left\|1-\sigma^{\prime}\right\| \cdot\left\|x^{\prime}-y^{\prime}\right\|+\gamma(0) L_{f}\|x-y\|_{1} \\
& +\left[\|\gamma\|_{L^{1}\left(\mathbb{R}^{+}\right)}+\left\|\gamma_{1}\right\|_{L^{1}\left(\mathbb{R}^{+}\right)}+\left\|\gamma_{2}\right\|_{L^{1}\left(\mathbb{R}^{+}\right)}\right] L_{f}\|x-y\|_{1} \\
\leq & \left(\|\alpha\|+\left\|\alpha^{\prime}\right\|+\|\alpha\| \cdot\left\|1-\sigma^{\prime}\right\|+\left[\gamma(0)+\|\gamma\|_{L^{1}\left(\mathbb{R}^{+}\right)}+\left\|\gamma_{1}\right\|_{L^{1}\left(\mathbb{R}^{+}\right)}+\left\|\gamma_{2}\right\|_{L^{1}\left(\mathbb{R}^{+}\right)}\right] L_{f}\right)\|x-y\|_{1} .
\end{aligned}
$$

By the assumptions, $\|\alpha\|+\left\|\alpha^{\prime}\right\|+\|\alpha\| \cdot\left\|1-\sigma^{\prime}\right\|+\left[\gamma(0)+\|\gamma\|_{L^{1}\left(\mathbb{R}^{+}\right)}+\left\|\gamma_{1}\right\|_{L^{1}\left(\mathbb{R}^{+}\right)}+\left\|\gamma_{2}\right\|_{L^{1}\left(\mathbb{R}^{+}\right)}\right] L_{f}<1$. Then, we deduce that Eq. 1.5 has a unique almost periodic solution.

Example 2.4. Let $\alpha(t) \equiv \frac{1}{6}, \sigma(t)=1+\sin t, \beta(t, s)=e^{-s^{2}}\left(\frac{\sin t+\sin \pi t)}{2}\right.$, and

$$
f(t, x, y)=\frac{1+\cos ^{2} \sqrt{2} t+\cos ^{2} t}{3} \cdot\left(\sin \frac{x}{18} \cos \frac{y}{18}+1\right) \text {. }
$$

It is easy to see that (H1) and (H2) hold with

$$
\gamma(s)=e^{-s^{2}}, \quad \gamma_{1}(s)=\left(\frac{1+\pi}{2}\right) e^{-s^{2}}, \quad \gamma_{2}(s)=2 s e^{-s^{2}} .
$$

Moreover, we have

$$
\begin{aligned}
\left|f\left(t, x_{1}, y_{1}\right)-f\left(t, x_{2}, y_{2}\right)\right| & \leq\left|\sin \frac{x_{1}}{18} \cos \frac{y_{1}}{18}-\sin \frac{x_{2}}{18} \cos \frac{y_{1}}{18}\right|+\left|\sin \frac{x_{2}}{18} \cos \frac{y_{1}}{18}-\sin \frac{x_{2}}{18} \cos \frac{y_{2}}{18}\right| \\
& \leq \frac{\left|x_{1}-x_{2}\right|+\left|y_{1}-y_{2}\right|}{18},
\end{aligned}
$$

which means that (H3) holds with $L_{f}=\frac{1}{18}$. By a direct calculation, we have

$$
\|\alpha\|=\frac{1}{6}, \quad\left\|\alpha^{\prime}\right\|=0, \quad\left\|1-\sigma^{\prime}\right\|=2, \quad \gamma(0)=1,
$$

and

$$
\|\gamma\|_{L^{1}\left(\mathbb{R}^{+}\right)}=\frac{\sqrt{\pi}}{2}, \quad\left\|\gamma_{1}\right\|_{L^{1}\left(\mathbb{R}^{+}\right)}=\frac{1+\pi}{4} \sqrt{\pi}, \quad\left\|\gamma_{2}\right\|_{L^{1}\left(\mathbb{R}^{+}\right)}=1
$$


which yields

$$
\|\alpha\|+\left\|\alpha^{\prime}\right\|+\|\alpha\| \cdot\left\|1-\sigma^{\prime}\right\|+\left[\gamma(0)+\|\gamma\|_{L^{1}\left(\mathbb{R}^{+}\right)}+\left\|\gamma_{1}\right\|_{L^{1}\left(\mathbb{R}^{+}\right)}+\left\|\gamma_{2}\right\|_{L^{1}\left(\mathbb{R}^{+}\right)}\right] L_{f}<1 .
$$

Then, by Theorem 2.3 , the following integral equation

$$
x(t)=\frac{1}{6} x(t-1-\sin t)+\int_{-\infty}^{t} \frac{\left(1+\cos ^{2} \sqrt{2} s+\cos ^{2} s\right)(\sin t+\sin \pi t)}{6} e^{-(t-s)^{2}} \cdot\left[\sin \frac{x(s)}{18} \cos \frac{x^{\prime}(s)}{18}+1\right] d s
$$

has a unique almost periodic solution.

\section{Pseudo almost periodic case}

In this section, we study the existence and uniqueness of pseudo almost periodic solution for Eq. 1.5. Set

$$
P A P^{1}(\mathbb{R})=\left\{f \in P A P(\mathbb{R}): f^{\prime} \in P A P(\mathbb{R})\right\},
$$

and

$$
B^{*}=\left\{\varphi \in P A P^{1}(\mathbb{R}): \varphi(t) \text { and } \varphi^{\prime}(t) \text { are uniformly continuous on } \mathbb{R}\right\} .
$$

It is easy to see that for every $\varphi \in P A P^{1}(\mathbb{R}), \varphi$ is uniformly continuous on $\mathbb{R}$ since $\varphi^{\prime}$ is bounded. In addition, we also equip $B^{*}$ with the norm $\|f\|_{1}=\|f\|+\left\|f^{\prime}\right\|$. For more details about $P A P^{1}(\mathbb{R})$, we refer the reader to [7].

Before stating the main result, we need recall some lemmas.

Lemma $3.1([1])$. Let $\beta: \mathbb{R} \times \mathbb{R}^{+} \rightarrow \mathbb{R}$ such that $t \rightarrow \beta(t, \cdot)$ is in $P A P\left(L^{1}\left(\mathbb{R}^{+}\right)\right)$and there exists $b \in L^{1}\left(\mathbb{R}^{+}\right)$ such that $\left|\beta^{\text {ap }}(t, s)\right| \leq b(s)$ for all $t \in \mathbb{R}$ and a.e. $s \in \mathbb{R}^{+}$, where $t \rightarrow \beta^{\text {ap }}(t, \cdot)$ is the almost periodic component of $t \rightarrow \beta(t, \cdot)$. Then $f \in A P(\mathbb{R})$ implies that $F \in A P(\mathbb{R})$, where

$$
F(t)=\int_{-\infty}^{t} \beta(t, t-s) f(s) d s, \quad t \in \mathbb{R} .
$$

Remark 3.2. In Lemma 3.1, if there exists $b \in L^{1}\left(\mathbb{R}^{+}\right)$such that $|\beta(t, s)| \leq b(s)$ for all $t \in \mathbb{R}$ and a.e. $s \in \mathbb{R}^{+}$, the conclusion is also true. In fact, noting that

$$
\left\{\beta^{a p}(t, \cdot): t \in \mathbb{R}\right\} \subset \overline{\{\beta(t, \cdot): t \in \mathbb{R}\}}{ }^{L^{1}\left(\mathbb{R}^{+}\right)} .
$$

Thus, for every $t \in \mathbb{R}$, there exists $\left\{t_{n}\right\} \subset \mathbb{R}$ such that

$$
\beta\left(t_{n}, \cdot\right) \rightarrow \beta^{a p}(t, \cdot) \text { in } L^{1}\left(\mathbb{R}^{+}\right),
$$

which yields that there is a subsequence $\left\{t_{k}\right\} \subset\left\{t_{n}\right\}$ such that

$$
\beta\left(t_{k}, s\right) \rightarrow \beta^{a p}(t, s) \text { a.e. } s \in \mathbb{R}^{+} .
$$

Then, we conclude that $\left|\beta^{a p}(t, s)\right| \leq b(s)$ for all $t \in \mathbb{R}$ and a.e. $s \in \mathbb{R}^{+}$.

Lemma 3.3. $B^{*}$ is also a Banach space with the norm $\|\cdot\|_{1}$.

Proof. Firstly, $P A P^{1}(\mathbb{R})$ is a Banach space under the norm $\|\cdot\|_{1}$ (cf. [7]). Secondly, it is not difficult to show that $B^{*}$ is closed in $P A P^{1}(\mathbb{R})$. This completes the proof.

Lemma 3.4 $([13])$. Let $\varphi \in P A P(\mathbb{R})$ be uniformly continuous on $\mathbb{R}$ and $\sigma \in P A P(\mathbb{R})$. Then $\varphi(\cdot-\sigma(\cdot)) \in$ $P A P(\mathbb{R})$. 
Theorem 3.5. Let $(\mathrm{H} 2)-(\mathrm{H} 3)$ and

$\left(\mathrm{H}^{\prime}\right) \alpha, \sigma \in B^{*}, f \in P A P\left(\mathbb{R} \times \mathbb{R}^{2}, \mathbb{R}\right)$ satisfies that $f(\cdot, x, y)$ is uniformly continuous on $\mathbb{R}$ uniformly for $(x, y)$ in any compact subset of $\mathbb{R}^{2}$, and $\beta: \mathbb{R} \times \mathbb{R}^{+} \rightarrow \mathbb{R}$ satisfies $\beta(\cdot, 0) \in B^{*}$, and $t \rightarrow \beta(t, \cdot)$ is in $P A P\left(L^{1}\left(\mathbb{R}^{+}\right)\right)$. Moreover, $t \rightarrow \beta_{i}(t, \cdot), i=1,2$, are both in $P A P\left(L^{1}\left(\mathbb{R}^{+}\right)\right)$and uniformly continuous on $\mathbb{R}$.

hold. Then, Eq. (1.5) has a unique pseudo almost solution in $B^{*}$ provided that

$$
\|\alpha\|+\left\|\alpha^{\prime}\right\|+\|\alpha\| \cdot\left\|1-\sigma^{\prime}\right\|+\left[\gamma(0)+\|\gamma\|_{L^{1}\left(\mathbb{R}^{+}\right)}+\left\|\gamma_{1}\right\|_{L^{1}\left(\mathbb{R}^{+}\right)}+\left\|\gamma_{2}\right\|_{L^{1}\left(\mathbb{R}^{+}\right)}\right] L_{f}<1 .
$$

Proof. Similar to the proof of Theorem 2.3 , we define $T: B^{*} \rightarrow C(\mathbb{R})$ by

$$
T x(t)=\alpha(t) x(t-\sigma(t))+\int_{-\infty}^{t} \beta(t, t-s) f\left(s, x(s), x^{\prime}(s)\right) d s, t \in \mathbb{R} .
$$

Next, let us show $T\left(B^{*}\right) \subset B^{*}$. Fix $x \in B^{*}$. By Lemma 3.4, we have

$$
x(\cdot-\sigma(\cdot)), \quad x^{\prime}(\cdot-\sigma(\cdot)) \in P A P(\mathbb{R}) .
$$

By Lemma 1.7, $t \rightarrow f\left(t, x(t), x^{\prime}(t)\right)$ belongs to $P A P(\mathbb{R})$. Then, it follows from Lemma 3.1 that

$$
t \rightarrow \int_{-\infty}^{t} \beta(t, t-s) f\left(s, x(s), x^{\prime}(s)\right) d s
$$

belongs to $P A P(\mathbb{R})$, and thus $T x \in P A P(\mathbb{R})$.

Recalling that

$$
\begin{aligned}
(T x)^{\prime}(t)= & \alpha^{\prime}(t) x(t-\sigma(t))+\alpha(t)\left(1-\sigma^{\prime}(t)\right) x^{\prime}(t-\sigma(t))+\beta(t, 0) f\left(t, x(t), x^{\prime}(t)\right) \\
& +\int_{-\infty}^{t} \beta_{1}(t, t-s) f\left(s, x(s), x^{\prime}(s)\right) d s+\int_{-\infty}^{t} \beta_{2}(t, t-s) f\left(s, x(s), x^{\prime}(s)\right) d s .
\end{aligned}
$$

Then, similar to the above proof, it is not difficult to show that $(T x)^{\prime} \in P A P(\mathbb{R})$.

It remains to show that $(T x)^{\prime}$ is uniformly continuous on $\mathbb{R}$. Since $x, \sigma$ are uniformly continuous on $\mathbb{R}$, it is not difficult to show that $x\left(\cdot-\sigma(\cdot)\right.$ and $x^{\prime}(\cdot-\sigma(\cdot))$ are uniformly continuous on $\mathbb{R}$. Noting that $x \in B^{*}$, $f(\cdot, x, y)$ is uniformly continuous on $\mathbb{R}$ uniformly for $(x, y)$ in any compact subset of $\mathbb{R}^{2}$, and

$$
\begin{aligned}
\mid f\left(t, x(t), x^{\prime}(t)-f\left(s, x(s), x^{\prime}(s) \mid \leq\right.\right. & \mid f\left(t, x(t), x^{\prime}(t)-f\left(s, x(t), x^{\prime}(t) \mid\right.\right. \\
& +L_{f}\left(|x(t)-x(s)|+\left|x^{\prime}(t)-x^{\prime}(s)\right|\right) .
\end{aligned}
$$

We conclude that $f\left(t, x(t), x^{\prime}(t)\right)$ is uniformly continuous on $\mathbb{R}$. Combining the above proof with $\left(\mathrm{H} 1^{\prime}\right)$, $\alpha^{\prime}(t) x(t-\sigma(t))+\alpha(t)\left(1-\sigma^{\prime}(t)\right) x^{\prime}(t-\sigma(t))+\beta(t, 0) f\left(t, x(t), x^{\prime}(t)\right)$ is uniformly continuous on $\mathbb{R}$.

We claim that $\int_{-\infty}^{t} \beta_{1}(t, t-s) f\left(s, x(s), x^{\prime}(s)\right) d s$ is also uniformly continuous on $\mathbb{R}$. In fact, we have

$$
\begin{aligned}
\mid \int_{-\infty}^{t_{1}} & \beta_{1}\left(t_{1}, t_{1}-s\right) f\left(s, x(s), x^{\prime}(s)\right) d s-\int_{-\infty}^{t_{2}} \beta_{1}\left(t_{2}, t_{2}-s\right) f\left(s, x(s), x^{\prime}(s)\right) d s \mid \\
= & \left|\int_{0}^{+\infty} \beta_{1}\left(t_{1}, s\right) f\left(t_{1}-s, x\left(t_{1}-s\right), x^{\prime}\left(t_{1}-s\right)\right) d s-\int_{0}^{+\infty} \beta_{1}\left(t_{2}, s\right) f\left(t_{2}-s, x\left(t_{2}-s\right), x^{\prime}\left(t_{2}-s\right)\right) d s\right| \\
\leq & \int_{0}^{+\infty} \gamma_{1}(s) \cdot\left|f\left(t_{1}-s, x\left(t_{1}-s\right), x^{\prime}\left(t_{1}-s\right)\right)-f\left(t_{2}-s, x\left(t_{2}-s\right), x^{\prime}\left(t_{2}-s\right)\right)\right| d s \\
& +\int_{0}^{+\infty}\left|\beta_{1}\left(t_{1}, s\right)-\beta_{1}\left(t_{2}, s\right)\right| \cdot\left\|f\left(\cdot, x(\cdot), x^{\prime}(\cdot)\right)\right\| \\
= & \int_{0}^{+\infty} \gamma_{1}(s) \cdot\left|f\left(t_{1}-s, x\left(t_{1}-s\right), x^{\prime}\left(t_{1}-s\right)\right)-f\left(t_{2}-s, x\left(t_{2}-s\right), x^{\prime}\left(t_{2}-s\right)\right)\right| d s \\
& +\left\|\beta_{1}\left(t_{1}, \cdot\right)-\beta_{1}\left(t_{2}, \cdot\right)\right\|_{L^{1}\left(\mathbb{R}^{+}\right)} \cdot\left\|f\left(\cdot, x(\cdot), x^{\prime}(\cdot)\right)\right\| .
\end{aligned}
$$


Then the conclusion follows from the uniform continuity of $t \rightarrow \beta_{1}(t, \cdot)$ and $f\left(t, x(t), x^{\prime}(t)\right)$. Similarly, one can show that $\int_{-\infty}^{t} \beta_{2}(t, t-s) f\left(s, x(s), x^{\prime}(s)\right) d s$ is uniformly continuous on $\mathbb{R}$. Thus, $(T x)^{\prime}$ is uniformly continuous on $\mathbb{R}$, and $T x \in B^{*}$.

The rest of proof is similar to that of Theorem 2.3. This completes the proof.

Next, by modifying Example 2.4, we give a simple example, which does not aim at generality.

Example 3.6. Let $\alpha(t) \equiv \frac{1}{6}, \sigma(t)=1+\sin t, \beta(t, s)=\frac{e^{-s^{2}}\left(\sin t+\sin \pi t+\frac{1}{1+t^{2}}\right)}{3}$ and

$$
f(t, x, y)=\frac{1+\cos ^{2} \sqrt{2} t+\cos ^{2} t+e^{-t^{2}}}{4} \cdot\left(\sin \frac{x}{18} \cos \frac{y}{18}+1\right) .
$$

Then, it is not difficult to show that $\left(\mathrm{H} 1^{\prime}\right),(\mathrm{H} 2)$ and (H3) hold.

\section{Application to differential equations}

In this section, stimulated by the idea in [1], we investigate the existence of almost periodic solutions for the following nonautonomous differential equation:

$$
x^{\prime}(t)=-a(t) x(t)+f\left(t, x(t), x^{\prime}(t)\right), \quad t \in \mathbb{R},
$$

where $a \in A P(\mathbb{R})$ with $\tilde{a}=\inf _{t \in \mathbb{R}} a(t)>0$, and $f$ satisfies (H3). We have the following results about the existence of almost periodic solutions for Eq. (4.1):

Theorem 4.1. Eq. (4.1) admits a unique almost periodic solution provided that $f \in A P\left(\mathbb{R} \times \mathbb{R}^{2}, \mathbb{R}\right)$ and $(1+\tilde{a}+3\|a\|) L_{f}<\tilde{a}$.

Proof. Since $\tilde{a}=\inf _{t \in \mathbb{R}} a(t)>0$, Eq. (4.1) admits a unique almost periodic solution if and only if the following integral equation

$$
x(t)=\int_{-\infty}^{t} e^{-\int_{s}^{t} a(r) d r} f\left(s, x(s), x^{\prime}(s)\right) d s, \quad t \in \mathbb{R},
$$

has a unique almost periodic solution. It is easy to see that Eq. (4.2) is special case of Eq. (1.5) with $\alpha(t) \equiv 0$ and $\beta(t, s)=e^{-\int_{t-s}^{t} a(r) d r}$. We will check that all the assumptions of Theorem 2.3 hold. For (H1), since $\beta(t, 0) \equiv 1$, it remains to prove $t \rightarrow \beta(t, \cdot)$ is in $A P\left(L^{1}\left(\mathbb{R}^{+}\right)\right)$. In view of

$$
\begin{aligned}
\|\beta(t+p, \cdot)-\beta(t, \cdot)\|_{L^{1}\left(\mathbb{R}^{+}\right)} & =\int_{0}^{+\infty}|\beta(t+p, s)-\beta(t, s)| d s \\
& =\int_{0}^{+\infty}\left|e^{-\int_{t+p-s}^{t+p} a(r) d r}-e^{-\int_{t-s}^{t} a(r) d r}\right| d s \\
& =\int_{M}^{+\infty}\left|e^{-\int_{t+p-s}^{t+p} a(r) d r}-e^{-\int_{t-s}^{t} a(r) d r}\right| d s+\int_{0}^{M}\left|e^{-\int_{t+p-s}^{t+p} a(r) d r}-e^{-\int_{t-s}^{t} a(r) d r}\right| d s \\
& \leq \int_{M}^{+\infty} 2 e^{-\tilde{a} s} d s+\int_{0}^{M} 2 e^{-\tilde{a} s}\left|e^{-\int_{t-s}^{t}[a(r+p)-a(r)] d r}-1\right| d s \\
& \leq \frac{2 e^{-\tilde{a} M}}{\tilde{a}}+2 M \sup _{s \in[0, M]}\left|e^{-\int_{t-s}^{t}[a(r+p)-a(r)] d r}-1\right| \\
& \leq \frac{2 e^{-\tilde{a} M}}{\tilde{a}}+2 M e^{2 M\|a\|} M \cdot \sup _{r \in \mathbb{R}}|a(r+p)-a(r)|,
\end{aligned}
$$

where $M>0$ and $p \in \mathbb{R}$ are two arbitrary constant. Combining this with $a \in A P(\mathbb{R})$ and choosing sufficiently large $M>0$, one can show that $t \rightarrow \beta(t, \cdot)$ is in $A P\left(L^{1}\left(\mathbb{R}^{+}\right)\right)$. In addition, it is easy to see that for all $t, s \in \mathbb{R}$, there holds

$$
|\beta(t, s)| \leq e^{-\tilde{a} s}, \quad\left|\beta_{1}(t, s)\right| \leq 2\|a\| e^{-\tilde{a} s}, \quad\left|\beta_{2}(t, s)\right| \leq\|a\| e^{-\tilde{a} s} .
$$


Moreover, it is not difficult to show that $t \rightarrow \beta_{i}(t, \cdot), i=1,2$, are both in $A P\left(L^{1}\left(\mathbb{R}^{+}\right)\right)$. Then, we conclude that (H2) holds. Noting that $\alpha(t) \equiv 0$, it remains to check $\left[\gamma(0)+\|\gamma\|_{L^{1}\left(\mathbb{R}^{+}\right)}+\left\|\gamma_{1}\right\|_{L^{1}\left(\mathbb{R}^{+}\right)}+\left\|\gamma_{2}\right\|_{L^{1}\left(\mathbb{R}^{+}\right)}\right] L_{f}<1$. By a direct calculation, we get

$$
\gamma(0)=1, \quad\|\gamma\|_{L^{1}\left(\mathbb{R}^{+}\right)}=\frac{1}{\tilde{a}}, \quad\left\|\gamma_{1}\right\|_{L^{1}\left(\mathbb{R}^{+}\right)}=\frac{2\|a\|}{\tilde{a}}, \quad\left\|\gamma_{2}\right\|_{L^{1}\left(\mathbb{R}^{+}\right)}=\frac{\|a\|}{\tilde{a}}
$$

By the assumptions, we have

$$
(1+\tilde{a}+3\|a\|) L_{f}<\tilde{a},
$$

i.e.,

$$
\left[\gamma(0)+\|\gamma\|_{L^{1}\left(\mathbb{R}^{+}\right)}+\left\|\gamma_{1}\right\|_{L^{1}\left(\mathbb{R}^{+}\right)}+\left\|\gamma_{2}\right\|_{L^{1}\left(\mathbb{R}^{+}\right)}\right] L_{f}=\left[1+\frac{1}{\tilde{a}}+\frac{3\|a\|}{\tilde{a}}\right] L_{f}<1 .
$$

So, all the assumptions of Theorem 2.3 hold. This completes the proof.

\section{Acknowledgment}

Q.-F. Zou acknowledges support from the Graduate Innovation Fund of Jiangxi Province (YC2015-S144). H.-S. Ding acknowledges support from NSFC (11461034), the Program for Cultivating Young Scientist of Jiangxi Province (20133BCB23009), the NSF of Jiangxi Province (20143ACB21001), and the Foundation of Jiangxi Provincial Education Department (GJJ150342).

\section{References}

[1] E. Ait Dads, P. Cieutat, L. Lhachimi, Positive pseudo almost periodic solutions for some nonlinear infinite delay integral equations, Math. Comput. Modelling, 49 (2009), 721-739. 1, 1, 2.2, 3.1, 4

[2] E. Ait Dads, P. Cieutat, L. Lhachimi, Existence of positive almost periodic or ergodic solutions for some neutral integral equations, Differ. Integral Equ., 22 (2009), 1075-1096.

[3] E. Ait Dads, K. Ezzinbi, Existence of positive pseudo almost periodic solution for a class of functional equations arising in epidemic problems, Cybern. Syst. Anal., 30 (1994), 133-144.

[4] E. Ait Dads, K. Ezzinbi, Almost periodic solution for some neutral nonlinear integral equation, Nonlinear Anal., 28 (1997), 1479-1489. 1

[5] A. Bellour, E. Ait Dads, Periodic solutions for nonlinear neutral delay integro-differential equations, Electron. J. Differential Equations, 100 (2015), 1-9. 1, 1

[6] D. Bugajewski, G. M. N'Guérékata, On some classes of almost periodic functions in abstract spaces, Int. J. Math. Math. Sci., 61 (2004), 3237-3247. 1, $1,1.9$

[7] T. Diagana, Almost automorphic type and almost periodic type functions in abstract spaces, Springer, New York, (2013). $3,3.3$

[8] H.-S. Ding, Y.-Y. Chen, G. M. N'Guérékata, Existence of positive pseudo almost periodic solutions to a class of neutral integral equations, Nonlinear Anal., 74 (2011), 7356-7364. 1, 1

[9] H.-S. Ding, Y.-Y. Chen, G. M. N'Guérékata, $C^{n}$-almost periodic and almost periodic solutions for some nonlinear integral equations, Electron. J. Qual. Theory Differ. Equ., 6 (2012), 1-13. 1

[10] H. S.-Ding, G. M. N'Guérékata, A note on the existence of positive bounded solutions for an epidemic model, Appl. Math. Lett., 26 (2013), 881-885.

[11] K. Ezzinbi, M. A. Hachimi, Existence of positive almost periodic solutions of functional equations via Hilberts projective metric, Nonlinear Anal., 26 (1996), 1169-1176. 1

[12] A. Fink, J. Gatica, Positive almost periodic solutions of some delay integral equations, J. Difference Equ., 83 (1990), 166-178. 1

[13] B. Liu, Pseudo Almost Periodic Solutions for CNNs with Continuously Distributed Leakage Delays, Neural Process Lett., 42 (2015), 233-256. 3.4

[14] W. Long, H.-S. Ding, Positive almost automorphic solutions for some nonlinear delay integral equations, Electron. J. Differ. Equ., 57 (2008), 1-8. 1

[15] R. Nussbanum, A periodicity threshold theorem for some nonlinear integral equations, SIAM J. Math. Anal., 9 (1978), 356-376. 1

[16] R. Torrejón, Positive almost periodic solutions of a state-dependent delay nonlinear integral equation, Nonlinear Anal., 20 (1993), 1383-1416. 1

[17] H. L. Smith, On periodic solutions of a delay integral equation modelling epidemics, J. Math. Biol., 4 (1977), 69-80. 1

[18] C. Zhang, Almost Periodic Type Functions and Ergodicity, Kluwer Academic Publishers, Dordrecht, (2003). 1 